Article

\title{
Disposable Fluorescence Optical pH Sensor for Near Neutral Solutions
}

\author{
Luca Ferrari *, Luigi Rovati, Paola Fabbri and Francesco Pilati \\ Department of Engineering Enzo Ferrari, University of Modena and Reggio Emilia, \\ via Vignolese 905, Modena I-41125, Italy; E-Mails: luigi.rovati@unimore.it (L.R.); \\ paola.fabbri@unimore.it (P.F.); francesco.pilati@unimore.it (F.P.) \\ * Author to whom correspondence should be addressed; E-Mail: luca.ferrari@ unimore.it; \\ Tel.: +39-059-205-6109; Fax: +39-059-205-6129.
}

Received: 31 October 2012; in revised form: 17 December 2012 / Accepted: 17 December 2012 / Published: 28 December 2012

\begin{abstract}
The design, development and performance evaluation of a fluorescence-based $\mathrm{pH}$ sensor for on-line measurements is presented. The $p K_{a}$ of the sensing element has been calculated to be 7.9, thus the sensor is suitable for measurement of near neutral solutions. The sensor consists of a low-cost disposable polymer sensing probe, in contact with the solution under test, interrogated by an optoelectronic transduction system. The $\mathrm{pH}$ sensitive dye is based on fluorescein $O$-methacrylate, which has been covalently linked to a hydrogel matrix, realized through the use of HEMA (2-hydroxyethyl methacrylate), HDDA (1,6-hexanediol diacrylate) and PEGDA (polyethylene glycol diacrylate). The optical interrogation setup, together with the electronics, has been developed to acquire and process the fluorescence signal. The sensor works over a $\mathrm{pH}$ range between 6.5 and 9.0. In the range between 7.0 and 8.0, the sensor shows a linear behavior with a maximum linearity error of $5 \%$. Thanks to the good performance of the sensing element and transduction system, the short term drift of the reading (measured over $40 \mathrm{~min}$ ) is lower than $0.15 \%$. The measuring system also exhibits good performance in terms of response time and reproducibility.
\end{abstract}

Keywords: optical sensor; pH sensor; fluorescence sensor; hydrogel; polymer matrix 


\section{Introduction}

$\mathrm{pH}$ (Latin: pondus hidrogenii) is a commonly measured parameter of great interest in many application fields, such as environmental monitoring [1,2], bioprocessing [3] and biomedical diagnostics [4]. The measurement of $\mathrm{pH}$ is routinely performed using the glass electrode. Nevertheless, the electrochemical approach suffers from many drawbacks, such as electromagnetic interference, difficulty in miniaturisation, and limitations when measuring aqueous suspensions of organic matter or low-ionic-strength solutions. With respect to electrochemical sensors, optical $\mathrm{pH}$ sensors allow for a higher sensitivity and selectivity, due to the luminescence phenomena. Moreover, they are insensitive to electromagnetic interference, they do not need any reference electrode and they are suitable for remote and disposable sensing, which is typical of in-situ applications [5,6].

Nowadays, the fluorescence phenomena is widely exploited to realize optical sensors, due to the higher sensitivity and versatility with respect to other detection schemes. In particular, fluorescein is widely used due to its high molar absorptivity at the wavelength of the argon laser $(\lambda=488 \mathrm{~nm})$, high fluorescence quantum yield and $\mathrm{pH}$-dependent emission spectra [7].

Several optical fluorescence-based $\mathrm{pH}$ sensors consist of a solid matrix permeable to protons, containing a $\mathrm{pH}$ sensitive dye, which can be reached by the analyte. Chemical bonding between the dye and the matrix is necessary to avoid undesirable leaching effects. $\mathrm{pH}$ measurements are performed revealing reversible variations in the intensity or lifetime of the fluorescent indicator; polymers are widely used as immobilization matrices for $\mathrm{pH}$-sensitive fluorescent dyes, thanks to the possibility of molecular tailoring to control and tune proton absorption [8-12].

Nevertheless, although lots of $\mathrm{pH}$ sensors are commercially available at present, only few of them are suitable for the fabrication of disposable sensing elements [13]. In fact, the key aspect of a disposable sensor is a cheap and simple transduction mechanism, which converts the information of interest into a readable signal, so that it is possible to use the same optical and electronic instrumentation for different measurements changing only the cheap and disposable sensing element. Disposability is becoming a fundamental characteristic in life science sensors, where exams performed on different patients require always new and sterilized sensing elements in contact with biological tissues.

In this paper, we present a disposable fluorescence-based optical $\mathrm{pH}$ sensor for on-line measurements in near neutral solutions. In particular, in Section 2, the polymer sensing element, the optics and electronics are fully described. Section 3 deals with the characterization of the sensor and finally the results are discussed and conclusions are drawn in Section 4.

\section{Materials and Methods}

In the next sections, after a brief theoretical discussion, the developed measurement system is presented. It basically consists of three parts: (i) polymer sensing element in contact with the solution, (ii) optical reading head and (iii) front-end and signal elaboration electronics.

\subsection{Theoretical Background}

The development of the present sensor is based on the fluorometric determination of $\mathrm{pH}$ [14]. Following the absorption of a photon, the excited molecules can lose energy through non-radiative 
relaxation, emission of a photon or energy transfer to an acceptor. The reemitted photons usually possess less energy, so they are shifted to the red part of the spectrum. This property represents a great advantage, compared with the absorption spectroscopy, since it decreases the level of the shot noise, which is proportional to the square root of the light intensity. In fact, when the emission light is only observed, the signal-to-noise ratio is greatly improved. The sensor presented in this work makes use of a polymerizable fluorescein, namely fluorescein $O$-methacrylate, to detect $\mathrm{pH}$ changes in the measured fluorescence intensity. Fluorescein $O$-methacrylate is a new kind of $\mathrm{pH}$-sensitive fluorescent monomer characterized by an excitation spectrum centered at $\lambda=490 \mathrm{~nm}$ and an emission spectrum centered at $\lambda=520 \mathrm{~nm}$. Lots of optical $\mathrm{pH}$ sensors based on the dissociation equilibrium of fluorescent dyes are usually cross-sensitive to ionic strength. Nevertheless, fluorescein carries the least negative charges compared with other fluorescent indicators such as HPTS and carboxyfluorescein, which leads to a lower dependence on the ionic strength. Variations in ionic strength in the range from $50 \mathrm{mM}$ to $400 \mathrm{mM}$ causes a pH error of ca. $0.05 \mathrm{pH}$ [15]. This error is acceptable and therefore fluorescein is a suitable indicator for $\mathrm{pH}$ measurement in aqueous solutions. Fluorescein exhibits multiple, $\mathrm{pH}$ dependent ionic equilibria [7]. Both the phenol and carboxylic acid functional groups of fluorescein are almost completely ionized in aqueous solutions above $\mathrm{pH} 9.0$ (Figure 1). Acidification of the fluorescein dianion first protonates the phenol $\left(p K_{a} \approx 6.4\right)$ to yield the fluorescein monoanion, then the carboxylic acid $\left(p K_{a}<5.0\right)$ to produce the neutral species of fluorescein. Further acidification generates a fluorescein cation $\left(p K_{a} \approx 2.1\right.$ ). Only the monoanion and dianion of fluorescein are fluorescent, with quantum yields of 0.37 and 0.93 , respectively. A further equilibrium involves the formation of a colorless nonfluorescent lactone (Figure 1).

Figure 1. Ionization equilibria of fluorescein.

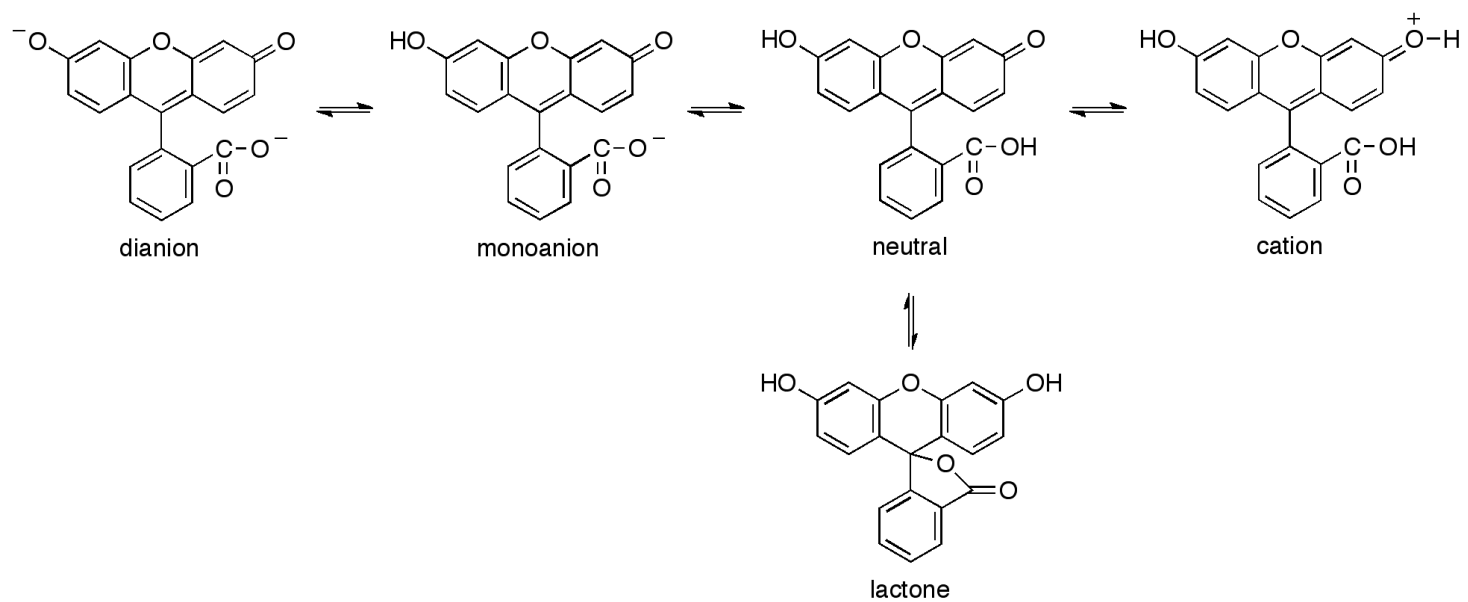

Nevertheless, the fluorescence emission spectrum of fluorescein, even in acidic solution, is dominated by the dianion, with only small contributions from the monoanion. Consequently, the wavelength and shape of the emission spectra resulting from excitation close to the dianion absorption peak at $490 \mathrm{~nm}$ are relatively independent of $\mathrm{pH}$, but the fluorescence intensity is dramatically reduced under acidic conditions. The mass-action law relationships between $\mathrm{pH}$ and fluorescence intensity determines the response curve of this sensing approach [16]. Considering a dye covalently linked to a polymer matrix, defining $I_{\max }$ and $I_{\min }$ as the fluorescence contribution of the fully deprotonated and the fully protonated 
form of the fluorescent indicator, respectively, the relation between the fluorescence emission intensity $I_{s}$ and $\mathrm{pH}$, in the presence of the sample under test, becomes [17-19]:

$$
p H=p K_{a}-b \cdot \log \left(\frac{I_{\max }-I_{s}}{I_{s}-I_{\min }}\right)
$$

where $p K_{a}$ is the acid-base constant of the indicator and $\mathrm{b}$ is a numerical coefficient, introduced to determine the slope of the function between $I_{\max }$ and $I_{\min }$. In fact, the chemical and physical properties of the matrix including the dye (e.g., polarity and viscosity) could affect its sensitivity near the $p K_{a}$, thus having different slopes for the same indicator in different matrices. Rewriting Equation (1) in terms of $I_{s}$ gives the well-known sigmoidal function [18]:

$$
I_{s}=\frac{I_{\max }+I_{\min } \cdot 10^{-\left(\frac{p H-p K_{a}}{b}\right)}}{1+10^{-\left(\frac{p H-p K a}{b}\right)}}=I_{\min }+\frac{I_{\max }-I_{\min }}{1+10^{-\left(\frac{p H-p K_{a}}{b}\right)}}
$$

This equation results in a nonlinear relationship between the fluorescence intensity versus $\mathrm{pH}$, which has been used for the calculation of the $p K_{a}$ of the dye included in the polymer matrix.

\subsection{Polymer Sensing Element}

The polymer sensing element in contact with the solution transduces the level of $\mathrm{pH}$ into an optical information; it consists of a polymer matrix and a $\mathrm{pH}$-sensitive dye covalently bonded to the polymer chains. The polymer matrix plays an important role because it is in direct contact with the solution and it contains the sensitive dye inside its molecular structure, so it has to be: (i) robust towards the flow, (ii) able to guarantee a fast penetration and mobility of the hydrogen ions, (iii) fast in response time, (iv) well adherent to the substrate of the measuring cell, (v) characterized by absence of or reduced dye leaching.

\subsubsection{Fabrication of the Polymer Matrix}

All chemicals needed for the realization of a hydrophilic, highly swellable polymer matrix containing an optical sensing element were of analytical grade, purchased from Sigma-Aldrich (Milan, Italy) and used without further purification. All aqueous solutions were prepared using distilled water. The matrix was prepared by the free-radical polymerization of 2-hydroxyethyl methacrylate (HEMA), whose thermodynamic affinity for water is well known [20], catalysed by the radical photoinitiator 4-(2-hydroxyethoxy)phenyl-(2-hydroxy-2-propyl)ketone (Irgacure 2959, BASF, Ludwigshafen, Germany), used at $1.0 \%$ wt. The polymerization was run in the presence of a tetrafunctional monomer, namely 1,6-hexanediol diacrylate (HDDA) added at 5.0\% wt with respect to HEMA, acting as crosslinker to avoid an excessive swelling degree of the acrylate matrix in contact with water, which could result in a poor mechanically resistance. Fluorescein O-methacrylate $97 \%$ (Figure 2(a)) was added to the acrylates mixture at $1.0 \%$ wt with respect to HEMA monomer; its methacrylate moiety allows for a covalent inclusion of the fluoresceine comonomer within the swellable polymer matrix, thus suppressing the severe drawback of indicator leaching. The covalent bonding between the HEMA matrix and the fluorescent dye is depicted in Figure 2(b). The overall swelling ratio of the polymer matrix in aqueous solutions was finely tuned by adding different amounts (up to 6.0\% wt) 
of a flexible macromer, namely poly(ethylene glycol) diacrylate (PEGDA, Ebecryl13, Cytec Industries, Woodland Park, NJ, USA), to further improve the polymer chain flexibility, and in turn ion mobility within the polymer matrix [21]. The mixture of chemicals was accurately mixed using a magnetic stirrer before being deposited by spin coating (500 rpm, $30 \mathrm{~s}$ ) onto a transparent PVC substrate (Figure 3(a)), and cured by UV-irradiation with a medium pressure mercury lamp under nitrogen with a light intensity on the surface of the sample of $30 \mathrm{~mW} / \mathrm{cm}^{2}$ for different time up to $70 \mathrm{~s}$.

Figure 3(b) reports a SEM micrograph of a cross-section of the sensing polymer film (thickness about $25 \mu \mathrm{m}$ ) deposited onto the PVC substrate (thickness $150 \mu \mathrm{m}$ ). Because of the manual realization of the sensing elements, it was difficult to firmly control the thickness of the polymer sensing layer; this disuniformity between different sensing elements may cause some variation in terms of response time and sensitivity. The polymer film uniformity could be improved through the application of instrumental deposition techniques, like the one reported by Tian et al. [22,23].

Figure 2. (a) Molecular structure of Fluorescein $O$-methacrylate. (b) Molecular structure of poly(HEMA) covalently bonded to Fluorescein $O$-methacrylate units.

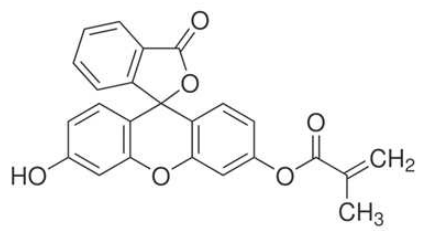

(a)

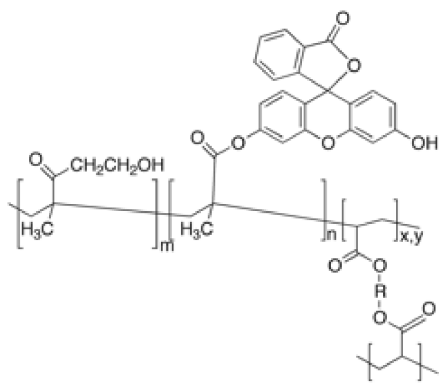

(b)
HDDA $\mathrm{R}=\left(-\mathrm{CH}_{2}\right)_{6}$

PEGDA R $=\left[\left(\mathrm{CH}_{2} \mathrm{CH}_{2} \mathrm{O}\right)_{\mathrm{P}} \mathrm{CH}_{2} \mathrm{CH}_{2}\right]$

Figure 3. (a) Top-view picture of the hydrophilic polymer matrix including the fluorescent indicator, used as the sensing element, excited by a blue LED. (b) SEM cross-section picture of the hydrophilic polymer matrix including the fluorescent indicator, used as the sensing element.

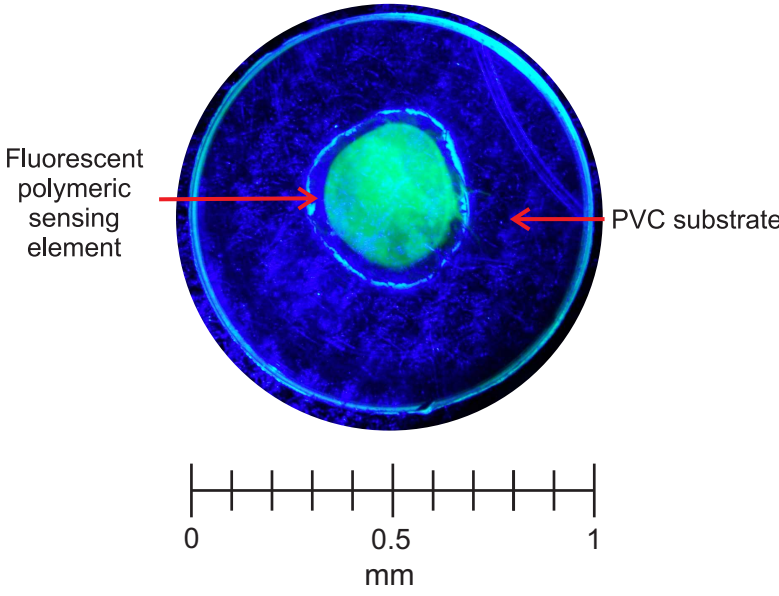

(a)

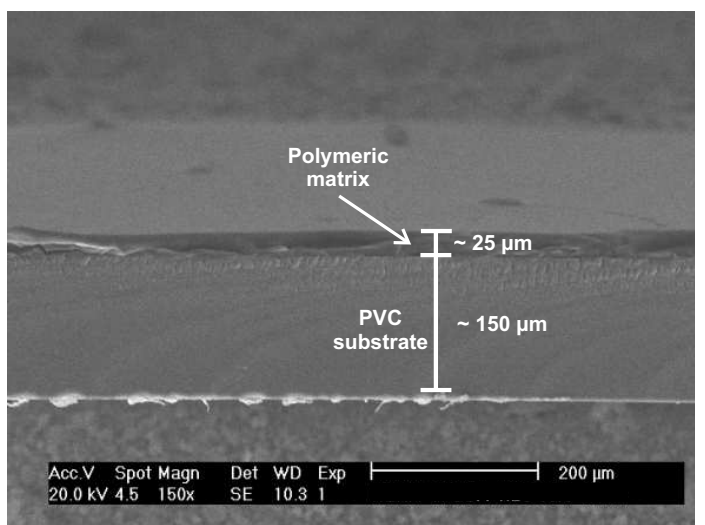

(b) 


\subsection{Optical Reading Head}

A schematic representation of the realized optical setup is reported in Figure 4. To perform fluorescence intensity variation measurements, it is necessary to excite the fluorescein; in the present work excitation was induced by a blue LED $\left(\lambda_{\text {peak }} \cong 480 \mathrm{~nm}\right)$, and the emitted fluorescence signal was collected. Since the optical power emitted by the LED is much higher than the emitted fluorescence, two filters inside the optical head have been positioned; in particular an excitation filter on the light emitted by the LED ( $\lambda_{\text {cutoff }}=485 \mathrm{~nm}$, ODL S.r.l., BG12, Bergamo, Italy $)$ and an emission filter ( $\Delta \lambda=515-535 \mathrm{~nm}$, Thorlabs, MF525-39, Munich, Germany) on the optical path of the fluorescence light towards the photodetector. The unwanted excitation light collected by the fluorescence channel produces an offset in the signal of interest comparable with the offsets introduced by the electronics. Thus, it can be compensated by a proper calibration of the sensor. Finally, two photodiodes have been used, one to collect the fluorescence signal and one to monitor the optical power emitted by the LED. The monitor photodiode is used to detect possible variations of the excitation optical power.

Figure 4. Schematic representation of the optical setup realized to perform fluorescence intensity measurements. It consists of a blue LED to excite the fluorescein, two optical filters (an excitation and an emission filter respectively) and finally two photodiodes, one to collect the fluorescence signal and one to monitor the optical power emitted by the LED.

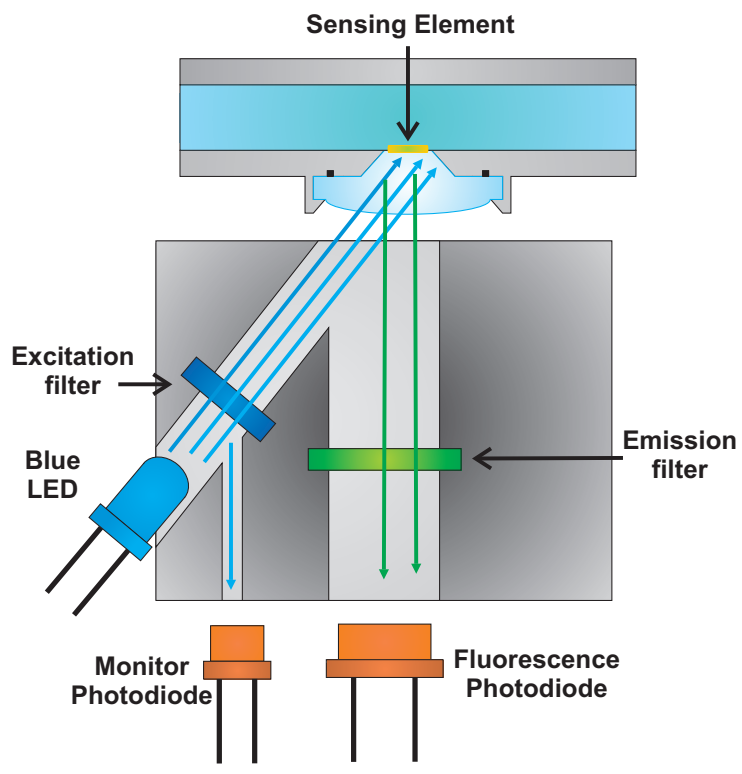

\subsection{Front-End and Signal Elaboration Electronics}

An electronic board has been developed to drive the LED, acquire and process the emitted fluorescence signal and the optical power emitted by the LED. In order to reduce the effect of photobleaching, which is typical in fluorescent indicators, the blue LED was excited by current pulses (duration $800 \mathrm{~ms}$ ) at a frequency of $0.125 \mathrm{~Hz}$ [24]. Moreover, to increase the robustness against the interferences and because of the low signal-to-noise ratio, the lock-in technique has been used, modulating sinusoidally the LED driving current during the pulse and demodulating the collected signal 
with the same signal (frequency $=6.06 \mathrm{kHz}$ ), as shown in Figure 5. In this way, only the signal of interest presents a non-zero average value, which is representative of the excitation and fluorescence intensities.

Figure 5. Schematic representation of the electronic board developed and realized to acquire and process the emitted fluorescence signal and the optical power emitted by the LED. The lock-in technique has been used, modulating the LED driving signal and demodulating the collected signal with the same sinusoidal signal. Only the signal of interest presents a non-zero average value.

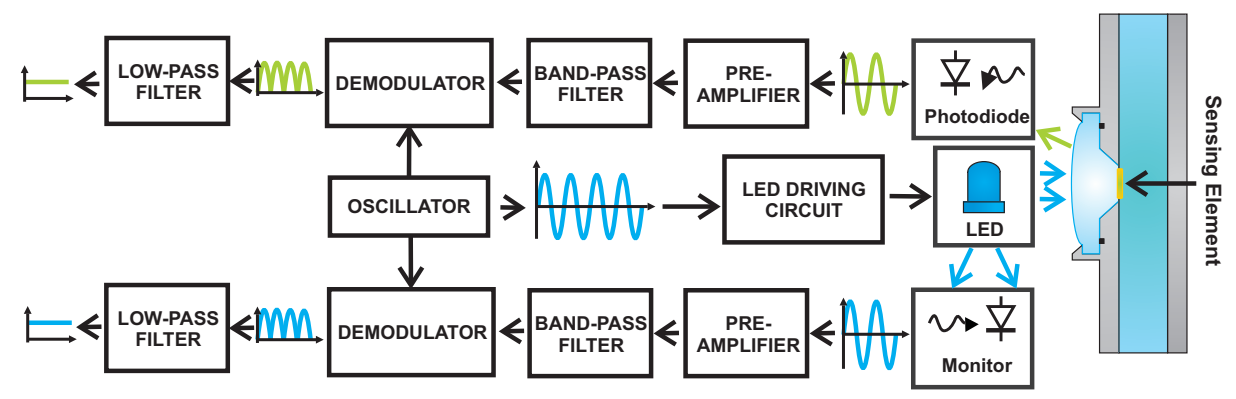

For the performed measurements, the fluorescence-to-monitor ratio was considered, thus avoiding that the variations in the optical power emitted by the LED could influence the fluorescence measurements. In fact, the fluorescence intensity $I_{s}$ is a function of the absorbed light [14], as reported in Equation (3):

$$
I_{s}=k I_{0} \phi \epsilon_{\lambda} l C
$$

where $I_{0}$ is the intensity of the exciting beam, $\phi$ is the quantum yield of the fluorophore, $\epsilon_{\lambda}$ is the molar absorptivity at $\lambda_{e x}, l$ is the optical path length in the sample, $C$ is the concentration of the fluorophore and $k$ is an instrumental factor. Considering the fluorescence-to-monitor ratio, it yields:

$$
I_{\text {Ratio }}=\frac{I_{s}}{I_{0}}=k \phi \epsilon_{\lambda} l C
$$

As shown in Equation (4), the ratio signal is independent from the optical power emitted by the LED. In the next sections of the article, it will be referred to the fluorescence-to-monitor ratio as $I_{\text {Ratio }}$ and to the normalized fluorescence-to-monitor ratio as $I_{\text {Norm }}$, defined as:

$$
I_{\text {Norm }}=\frac{I_{s}}{\left.I_{0} \cdot I_{\text {Ratio }}\right|_{\text {Max }}}
$$

\section{Experimental Results}

In order to test the sensor, a fluidic system has been realized. As shown in the schematic representation in Figure 6(a) and in the picture in Figure 6(b), it consists of (i) a closed loop circulator, (ii) the optical head with the sensing element inside, (iii) a beaker with a reference glass electrode $\mathrm{pH}$-meter (Eutech Instruments, XS pH 700, Nijkerk, The Netherlands) to measure the $\mathrm{pH}$ of the solution and a thermocouple to measure the temperature of the solution and correct the $\mathrm{pH}$ value.

All the measurements were performed at room temperature, with a flow rate of $200 \mathrm{~mL} / \mathrm{min}$. The $\mathrm{pH}$ of the solution was changed by adding acetic acid or ammonium hydroxide to the solution. 
Figure 6. (a) Schematic representation of the fluidic system used to test the $\mathrm{pH}$ sensor. It consists of (i) a closed loop circulator, (ii) the optical head with the sensing element inside, (iii) a beaker with a reference glass electrode $\mathrm{pH}$-meter to measure the $\mathrm{pH}$ of the solution and a thermocouple to measure the temperature of the solution and correct the $\mathrm{pH}$ value. (b) Picture of the fluidic system used to test the $\mathrm{pH}$ sensor.

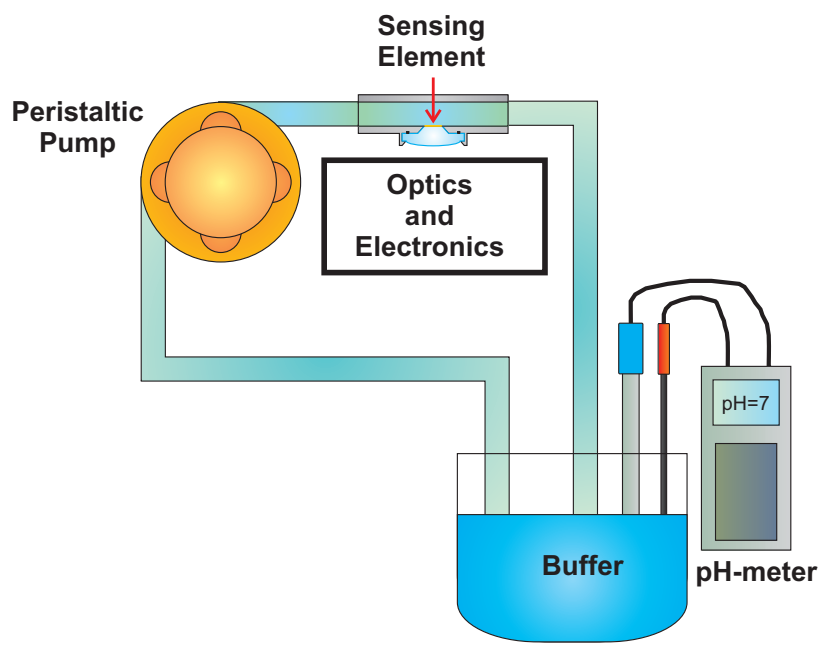

(a)

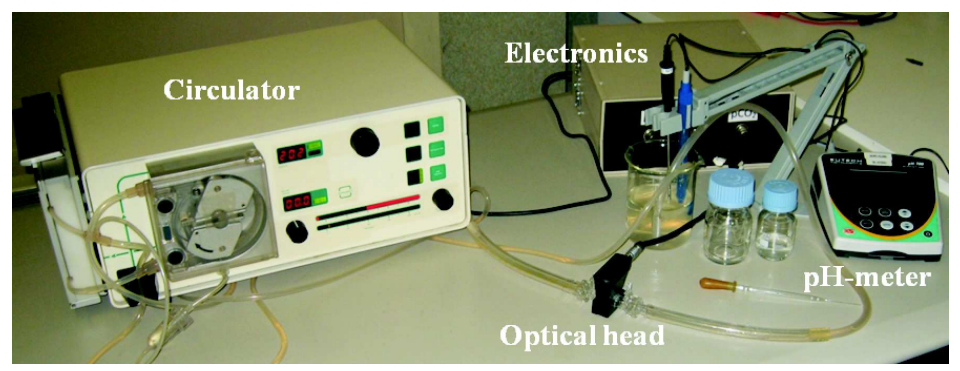

(b)

\subsection{Characterization of the Sensor $p K_{a}$}

A fluorometric titration has been performed to investigate the $p K_{a}$ of the indicator inside the polymer matrix in aqueous solutions. In Figure $7, I_{\text {Norm }}$ versus time acquired at different $\mathrm{pH}$ values is shown. The steady state $\mathrm{pH}$ values of the solution as measured by the reference instrument are reported close to the curve. In Figure 8, the average of the $I_{\text {Norm }}$ signal over a time interval of $60 \mathrm{~s}$ for every reference $\mathrm{pH}$ value is shown.

$I_{N o r m}$ values have been fitted to a sigmoidal function, represented in Figure 8 and determined by applying Equations (5) and (2), where $I_{\max }=1.98, I_{\min }=1$ and $b=1.10$. The $p K_{a}$ of the indicator was thus estimated to be 7.9. This value is bigger than the one reported in literature for the fluorescein in aqueous solutions, i.e., 6.4 [25]. According to Vasylevska et al. [18], an increase in $p K_{a}$ is observed upon covalent immobilization of the indicator and is attributed to the decrease in the polarity of the microenvironment.

The sigmoidal interpolation curve can be approximated with a linear curve for a range of values of $\mathrm{pH}$ close to the $p K_{a}$ of the indicator. In particular, here we propose to consider a linearity range between 7 and 8 . In the next section, the linearity characteristics of the sensor within this range are presented. 
Figure 7. $I_{\text {Norm }}$ versus time of a measurement performed in an aqueous solution, changing the $\mathrm{pH}$ value of the solution. The steady state $\mathrm{pH}$ values of the solution as measured by the reference instrument are reported close to the curve.

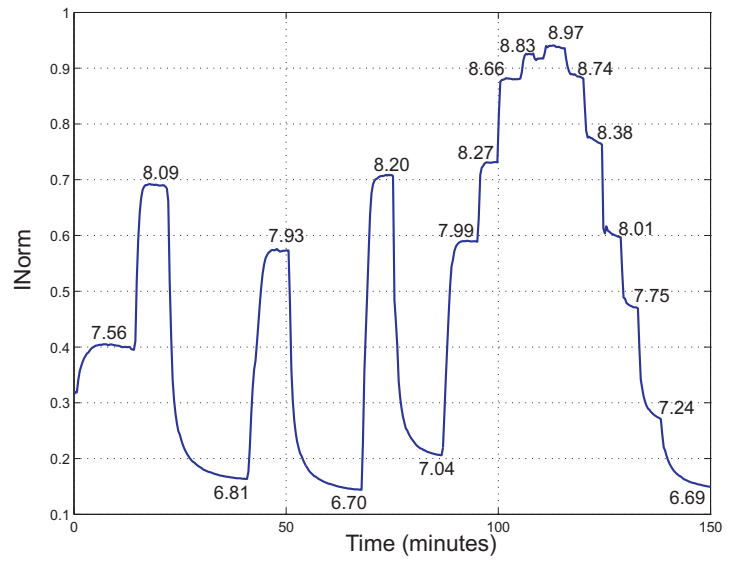

Figure 8. $I_{\text {Norm }}$ versus reference $\mathrm{pH}$ and sigmoidal interpolation curve.

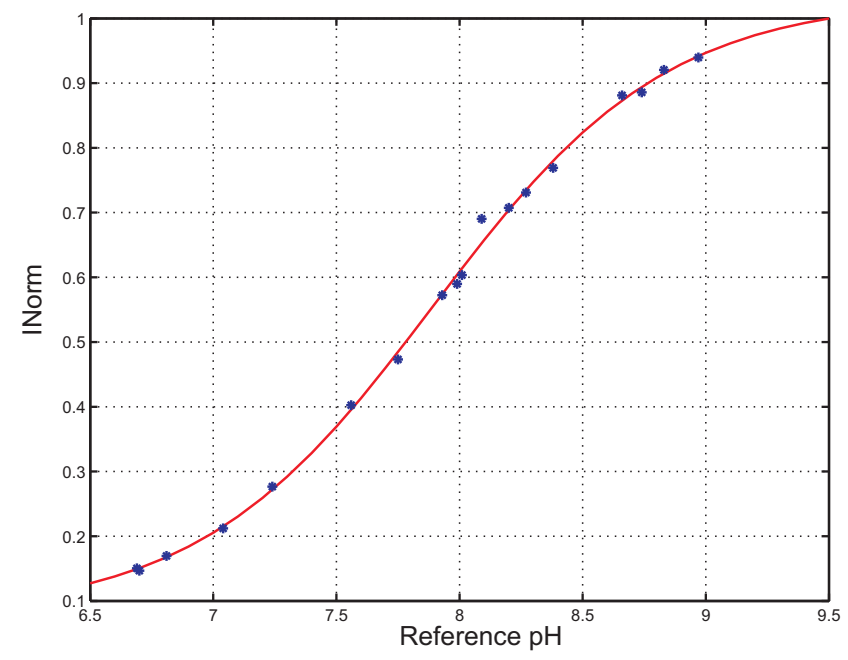

\subsection{Evaluation of the Non-Linearity Error}

In Figure $9, I_{\text {Norm }}$ versus time acquired at different $\mathrm{pH}$ values in the range $7.0-8.0$ is shown. It can be noted that the new normalization of $I_{\text {Ratio }}$ tends to expand and shift the ranges of values of $I_{\text {Norm }}$ respect to the data shown in Figure 7.

For every reference $\mathrm{pH}$ value, the average of $I_{\text {Norm }}$ has been calculated over an interval of $60 \mathrm{~s}$. Afterward, these values were fitted to a linear function as:

$$
\bar{I}_{\text {Norm }}=0.546 \cdot p H_{\text {Reference }}-3.285
$$

where $\bar{I}_{\text {Norm }}$ is the average value of $I_{\text {Norm }}$ and $p H_{\text {Reference }}$ is the $\mathrm{pH}$ measured by the reference $\mathrm{pH}$ electrode. Hence, the estimated $\mathrm{pH}$ values were calculated as:

$$
p H_{\text {Estimated }}=\frac{\bar{I}_{\text {Norm }}-q}{m}=\frac{\bar{I}_{\text {Norm }}+3.285}{0.546}
$$

In Figure 10(a) the estimated values versus reference $\mathrm{pH}$ are shown, together with the fitting line $(\mathrm{R}=0.9958)$, whereas Figure 10(b) shows the difference between the estimated and reference $\mathrm{pH}$ 
values, for every reference $\mathrm{pH}$ value. Thus, the Integral Non-linearity error, which is a parameter representing the maximum deviation between the sigmoidal interpolation curve of Equation (2) and the linear interpolation curve of Equation (6), was calculated to be 0.02 units of $\mathrm{pH}$, which represents the $2 \%$ in the range 7.0-8.0. Finally, the Integral Non-linearity error was calculated also for the measurement reported in Figure 7 and was determined to be 0.05 units of $\mathrm{pH}$, which represents the $5 \%$ in the range 7.0-8.0. These small variations between the Integral Non-linearity errors are probably due to different sensing elements used to perform these tests.

Figure 9. $I_{\text {Norm }}$ versus time acquired at different $\mathrm{pH}$ values in the range 7.0-8.0. The steady state $\mathrm{pH}$ values of the solution as measured by the reference instrument are reported close to the curve.

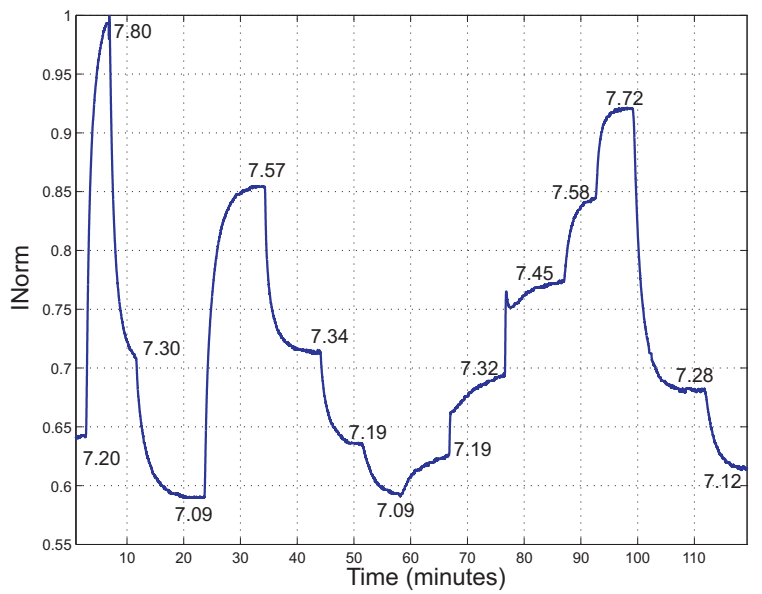

Figure 10. (a) Estimated versus reference $\mathrm{pH}$ and fitting line. (b) Difference between estimated and reference $\mathrm{pH}$ versus reference $\mathrm{pH}$.

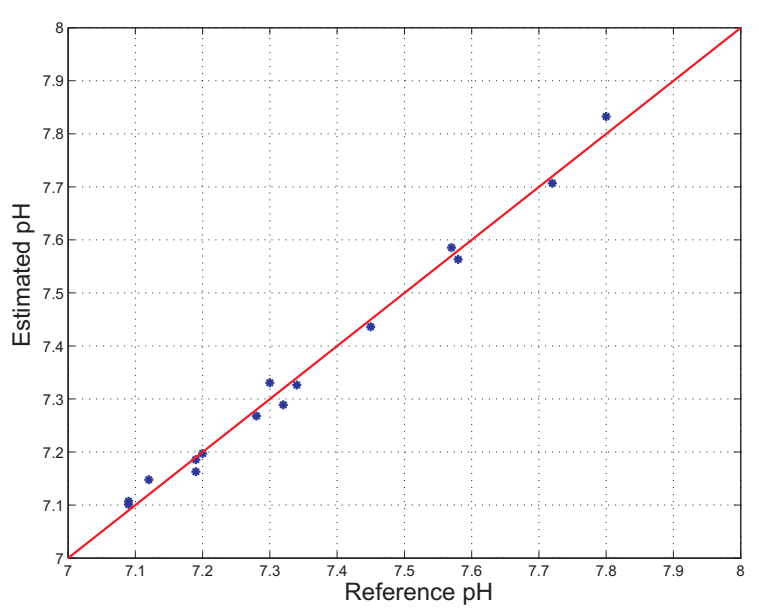

(a)

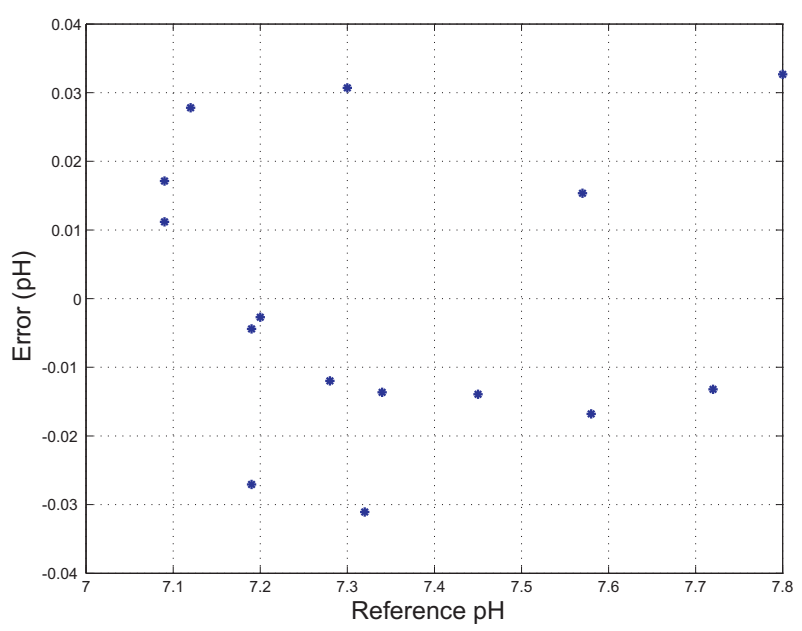

(b)

\subsection{Short Term Stability}

Figure 11(a) shows the estimated $\mathrm{pH}$ values, determined through Equation (7), versus time acquired firstly changing the $\mathrm{pH}$ of the solution and then keeping it constant. Assuming that the $\mathrm{pH}$ of the solution is completely stabilized at $\mathrm{t}=60 \mathrm{~min}$, the short term stability can be determined by evaluating the 
deviations of the estimated $\mathrm{pH}$ respect to its mean value in the time interval 60-100 min. As shown in Figure 11(b), no significant drifts have been observed in this time interval; all deviations are within $0.15 \%$ of the mean value of the estimated $\mathrm{pH}$.

Figure 11. (a) Estimated $\mathrm{pH}$ versus time of a measurement performed changing the $\mathrm{pH}$ value of the solution and then keeping it constant. The steady state $\mathrm{pH}$ values of the solution as measured by the reference instrument are reported close to the curve. (b) Deviations of the estimated $\mathrm{pH}$ respect to its mean value in the time interval 60-100 min. All deviations are within $0.15 \%$ of the mean value of the estimated $\mathrm{pH}$ (dashed lines).

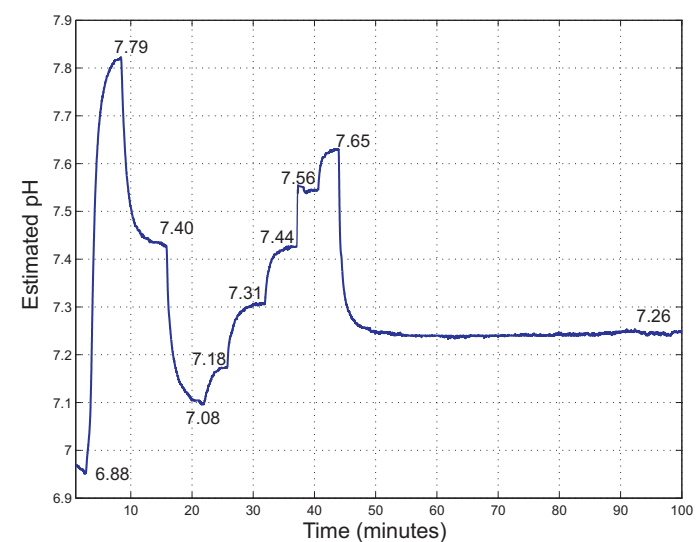

(a)

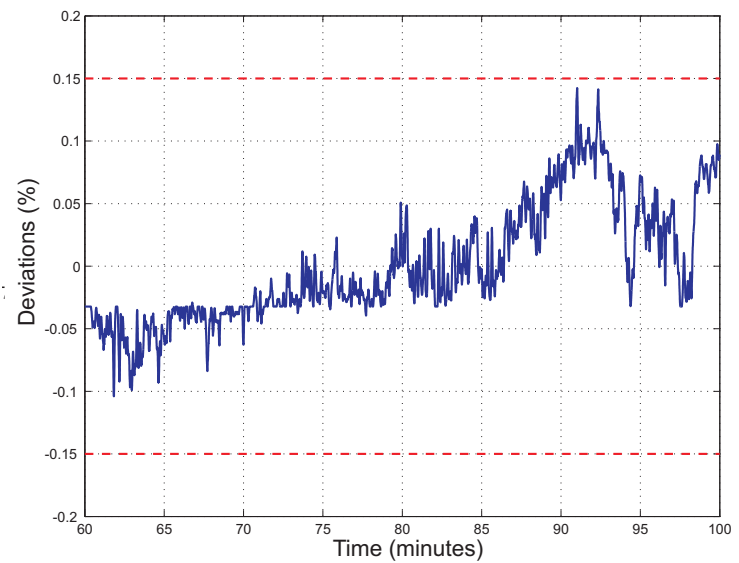

(b)

\subsection{Response Time}

The sensor response time, the so-called $\tau_{90}$, is defined as the time required for the sensor output to reach $90 \%$ of the change from its previous value to the final settled value. To measure the response time of the developed sensor, the fluidic system reported in Figure 12 has been realized.

The setup shown in Figure 6(a) has been integrated by a 3-way valve, thus allowing switching between two different buffer solutions (Fisher Scientific, Sigma-Aldrich). The measurement was performed by pumping the first buffer solution till the sensor stabilized, then switching to the second buffer solution and waiting for the stabilization of the sensor and finally switching back to the first one. The measurements were performed with the same sensing element (50 $\mu \mathrm{m}$ thick), at room temperature at a flow rate of $200 \mathrm{~mL} / \mathrm{min}$. The whole circuit volume was about $200 \mathrm{~mL}$. In Figure 13(a-c), $I_{\text {Norm }}$ versus time is reported for three different test conditions.

In Table 1, the calculated rise and fall times $\left(\tau_{90}\right)$ of the performed measurements are reported. The response time of the sensor depends on several factors, such as the flow-rate and the thickness of the film. Response times reported in Figure 13 are substantially different compared with those of Figures 7, 9 and 11(a). This large difference can be ascribed to the thickness of the sensing film. We have experimentally observed that even small differences in thickness can result in large changes in the dynamic response of the sensor. Moreover, the response time depends on the initial and final $\mathrm{pH}$ value. In fact, from the data reported in Table 1, it can be noticed that the response time is highly dependent on the $\mathrm{pH}$ values with respect to the $p K_{a}$ of the fluorescent dye. In fact, the response time is longer for the first variation of $\mathrm{pH},(\mathrm{pH}=7.0 \rightarrow 8.0$, Figure 13(a)), which has been done between 
$\mathrm{pH}$ values below or equal to the $p K_{a}\left(p K_{a} \approx 7.9\right)$. If the final $\mathrm{pH}$ value is much higher than the $p K_{a}$, the response time is significantly reduced; in fact, considering the second variation $(p H=8.0 \rightarrow 9.0$, Figure 13(b)), the response time is improved. Nevertheless, it can be noticed that, if the variation is done between two $\mathrm{pH}$ values chosen so that they are on either side of the $p K_{a}$ value, $(p H=7.0 \rightarrow 9.0$, Figure 13(c)), the rise and fall times are further improved ( 7 min per unit of $\mathrm{pH}$ and 13 min per unit of $\mathrm{pH}$, respectively). Finally, it can be noticed that, if the final $\mathrm{pH}$ value is higher than the $p K_{a}$, the rise time is lower than the fall time, whereas if the final $\mathrm{pH}$ value is lower than the $p K_{a}$, the fall time is lower than the rise time.

Figure 12. Schematic representation of the fluidic system used to measure the $\mathrm{pH}$ sensor response time. It consists of (i) a closed loop circulator, (ii) the optical head with the sensing element inside, (iii) a beaker with a glass electrode $\mathrm{pH}$-meter to measure the $\mathrm{pH}$ of the solution and a thermocouple to measure the temperature of the solution and correct the $\mathrm{pH}$ value. A 3-way valve has been added to switch between two different buffer solutions.

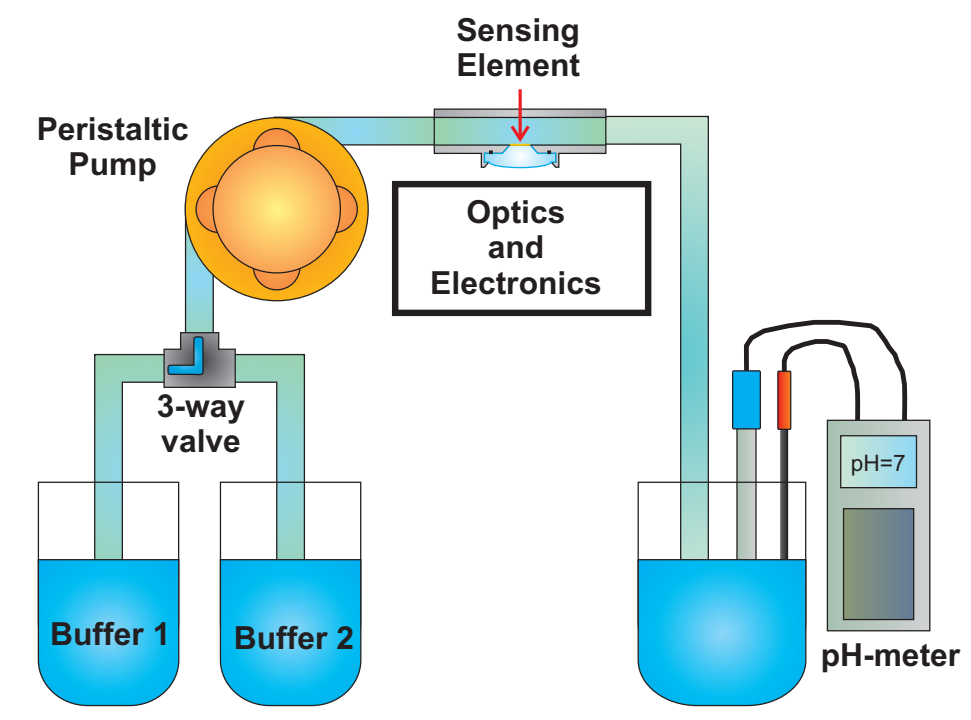

Table 1. Rise and fall times estimated during the three performed tests.

\begin{tabular}{lll}
\hline & Rise time $\tau_{90}(\min )$ & Fall time $\tau_{90}(\min )$ \\
\hline$p H=7.0 \rightarrow 8.0$ & 54.0 & 23.0 \\
$p H=8.0 \rightarrow 9.0$ & 9.5 & 17.0 \\
$p H=7.0 \rightarrow 9.0$ & 14.0 & 26.0 \\
\hline
\end{tabular}

Nevertheless, this dynamic behavior can be improved by using thinner polymer sensing elements. The thickness of the sensing element depends on a trade-off between the response time and the signal-to-noise ratio. As long as the signal-to-noise ratio of our sensor remains high enough, it is possible to use thinner polymer films and thus achieve shorter response time. 
Figure 13. $I_{\text {Norm }}$ versus time acquired during the three tests performed to evaluate the response time of the sensor and its dependency on the $\mathrm{pH}$ value. (a) Sensor response to a change in $\mathrm{pH}$ from 7 to 8 and back; (b) sensor response to a change in $\mathrm{pH}$ from 8 to 9 and back; (c) sensor response to a change in $\mathrm{pH}$ from 7 to 9 and back.

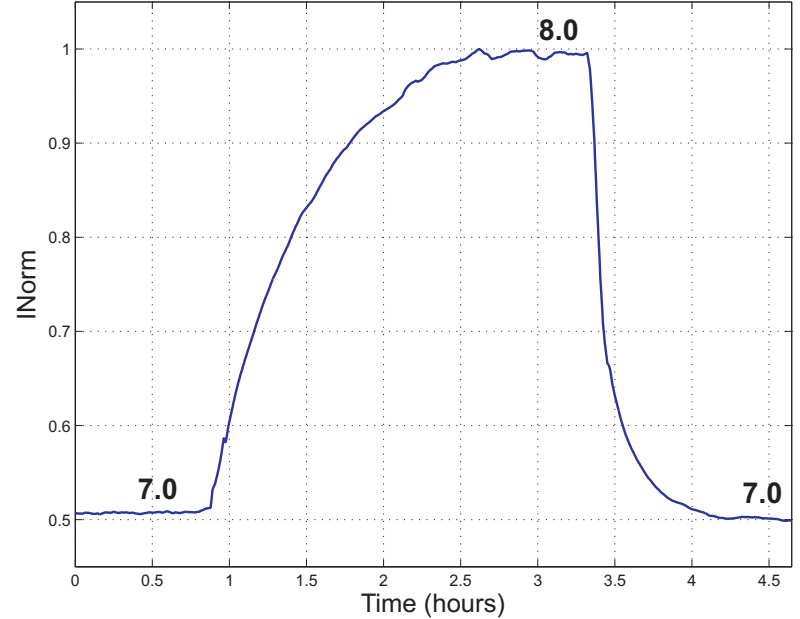

(a)

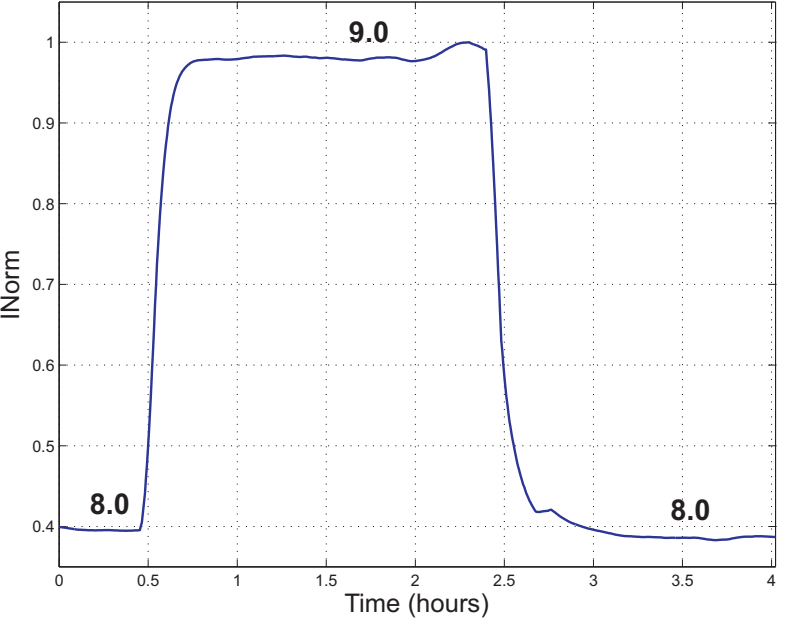

(b)

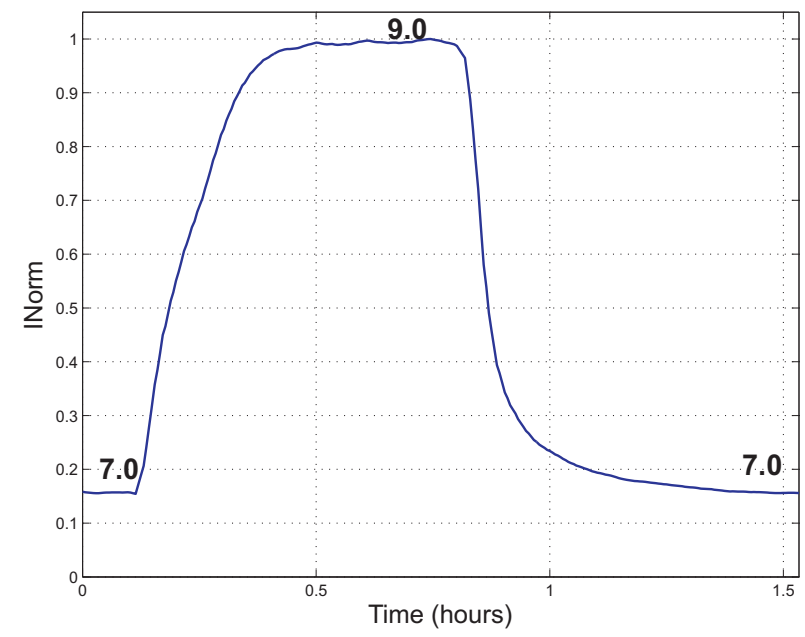

(c)

\subsection{Reproducibility}

The reproducibility of the $\mathrm{pH}$ sensor has been evaluated using the fluidic system represented in Figure 12, by changing the $\mathrm{pH}$ between the same two buffer solutions, i.e., 8.0 and 9.0 (Fisher, Sigma-Aldrich, Milan, Italy). In Figure 14(a), $I_{\text {Norm }}$ versus time is reported. The first test (blue) was performed the day before the second test (red), using the same polymer sensing element. In Figure 14(b) the mean values of the $I_{\text {Norm }}$ signal, measured at $p H=8.0$ and $p H=9.0$, are reported, together with the error bars.

The mean values were calculated considering the average values of $I_{\text {Norm }}$ in the steady-states at $\mathrm{pH}=8.0$ and $\mathrm{pH}=9.0$ respectively. The error bars represent the standard deviations; for the reference $\mathrm{pH}$ values they were determined through the uncertainty reported on the datasheet of the buffer solutions, i.e., $\mathrm{pH}=8.0 \pm 0.02$ and $\mathrm{pH}=9.0 \pm 0.02$ respectively, whereas for the $I_{\text {Norm }}$ signal they were determined by 
the sample standard deviation $(s)$. Finally, the mean values $\left(\bar{I}_{N o r m}\right)$ and the relative standard deviations $\left(\sigma=s / \bar{I}_{\text {Norm }}\right.$ ) of $I_{\text {Norm }}$ were determined to be $0.425 \pm 1.80 \%$ at $\mathrm{pH}=8.0$ and $0.993 \pm 0.60 \%$ at $\mathrm{pH}=9.0$.

Figure 14. (a) Acquired $I_{\text {Norm }}$ versus time changing the $\mathrm{pH}$ value between 8.0 and 9.0 to evaluate the reproducibility of the $\mathrm{pH}$ sensor. Two tests were performed (blue line and red line) on two consecutive days. (b) Mean values and error bars of the $I_{N o r m}$ signal, measured at $\mathrm{pH}=8.0$ and $\mathrm{pH}=9.0$.

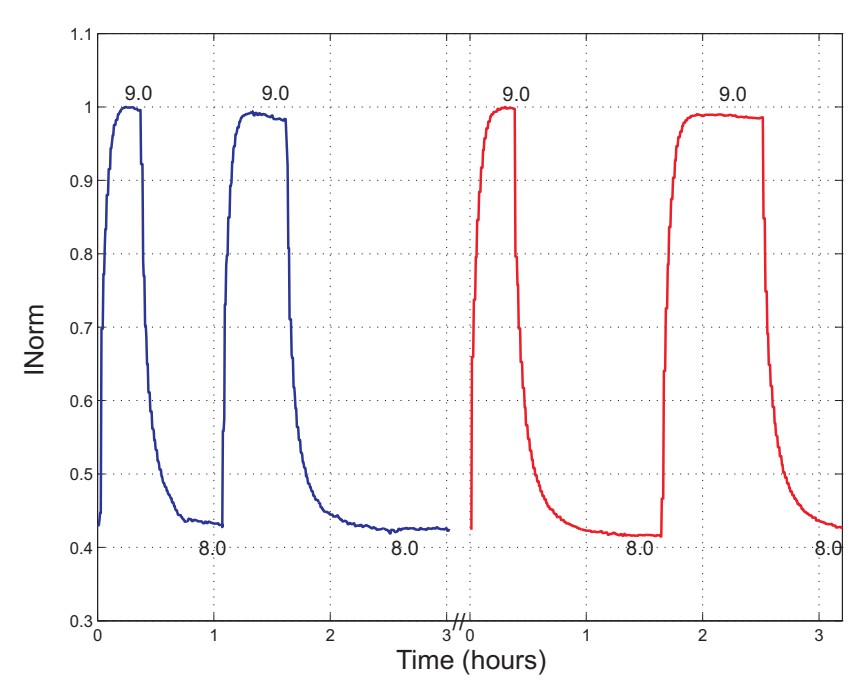

(a)

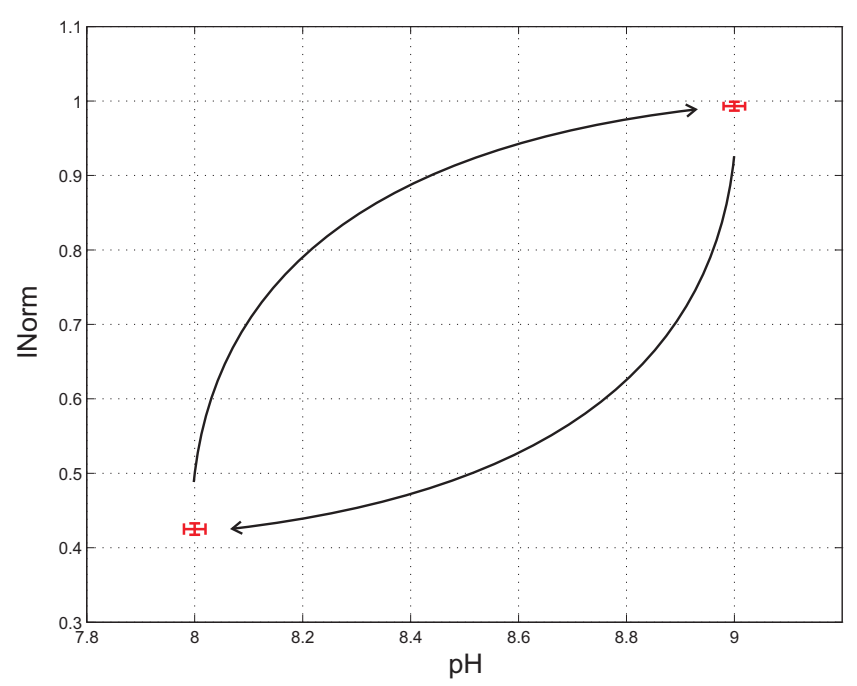

(b)

\section{Discussion and Conclusions}

A disposable optical fluorescence sensor has been developed. The sensor is based on the $\mathrm{pH}$-dependent fluorescence of a purposely developed polymer matrix including a fluorescent monomer (fluorescein $O$-methacrylate $97 \%$ ). This fluorescent monomer is covalently bonded to the hydrophilic polymer chains to realize a disposable sensing element without the risk of dye leaching. The optical head and the front-end electronics have been also developed to collect and process the fluorescence signal together with the electronics to acquire and process the signals of interest. Since the $p K_{a}$ of the sensing element has been calculated to be 7.9, the sensor is suitable for measurement of near neutral solutions. Good performance in terms of linearity (in the range 7.0-8.0), stability and reproducibility has been observed.

It has to be noticed that the response time strongly depends on the measuring conditions, i.e., amplitude of the $\mathrm{pH}$ range and its limit values. Obviously, this parameter also depends on the physico-chemical characteristics of the sensing element. In fact, it has been observed that the response time could change considerably for different sensing elements. This variation is mainly due to different thicknesses and cross-linking degrees of the sensing elements, caused by the manual realization. For a thickness of the sensing film of $50 \mu \mathrm{m}$, we measured a response time between $10 \mathrm{~min}$ and $1 \mathrm{~h}$. For many applications, this dynamic response may be sufficient; nevertheless it is always possible to reduce the thickness of the polymer film to improve the response time. Furthermore, it was observed that the response time diverges in the case of $\mathrm{pH}$ excursions that have their final value close to $p K_{a}$ of the sensing 
element. Therefore, to improve the sensor dynamic response, this value can be tuned by adjusting the sensing matrix to prevent, in the application of interest, this condition to occur.

Future work will focus on the realization of the sensing elements in order to diminish the performance spread and improve their uniformity.

\section{References}

1. Dybko, A.; Wroblewski, W.; Rozniecka, E.; Pozniak, K.; Maciejewski, J.; Romaniuk, R.; Brzozka, Z. Assessment of water quality based on multiparameter fiber optic probe. Sens. Actuators $B$ Chem. 1998, 51, 208-213.

2. Schirrmann, M.; Gebbers, R.; Kramer, E.; Seidel, J. Soil pH mapping with an on-the-go sensor. Sensors 2011, 11, 573-598.

3. Kermis, H.R.; Kostov, Y.; Harms, P.; Rao, G. Dual excitation ratiometric fluorescent pH sensor for noninvasive bioprocess monitoring: Development and application. Biotechnol. Progr. 2002, 18, 1047-1053.

4. Gannot, I.; Ron, I.; Hekmat, F.; Chernomordik, V.; Gandjbakhche, A. Functional optical detection based on pH dependent fluorescence lifetime. Lasers Surg. Med. 2004, 35, 342-348.

5. Lin, J. Recent development and applications of optical and fiber-optic $\mathrm{pH}$ sensors. Trends Anal. Chem. 2000, 19, 541-552.

6. Bilro, L.; Alberto, N.; Pinto, J.L.; Nogueira, R. Optical sensors based on plastic fibers. Sensors 2012, 12, 12184-12207.

7. Johnson, I.; Spence, M.T.Z. Molecular Probes Handbook, A Guide to Fluorescent Probes and Labeling Technologies, 11th ed.; Invitrogen Life Technologies: Carlsbad, CA, USA, 2010.

8. Xu, H.; Sadik, O.A. Design of a simple optical sensor for the detection of concentrated hydroxide ions in an unusual pH range. Analyst 2000, 125, 1783-1786.

9. Choi, M.F. Spectroscopic behaviour of 8-hydroxy-1,3,6-pyrenetrisulphonate immobilized in ethyl cellulose. J. Photochem. Photobiol. A Chem. 1997, 104, 207-212.

10. Richter, A.; Paschew, G.; Klatt, S.; Lienig, J.; Arndt, K.F.; Adler, H.J.P. Review on hydrogel-based pH sensors and microsensors. Sensors 2008, 8, 561-581.

11. Cajlakovic, M.; Lobnik, A.; Werner, T. Stability of new optical pH sensing material based on cross-linked poly(vinyl alcohol) copolymer. Anal. Chim. Acta 2002, 455, 207-213.

12. Wencel, D.; MacCraith, B.; McDonagh, C. High performance optical ratiometric sol-gel-based $\mathrm{pH}$ sensor. Sens. Actuators B Chem. 2009, 139, 208-213.

13. Rovati, L.; Fabbri, P.; Ferrari, L.; Pilati, F. Construction and evaluation of a disposable pH sensor based on a large core plastic optical fiber. Rev. Sci. Instrum. 2011, 82, doi:10.1063/1.3541795.

14. Kostov, Y.; Rao, G. Low-cost optical instrumentation for biomedical measurements. Rev. Sci. Instrum. 2000, 71, 4361-4374.

15. Weidgans, B.M. New Fluorescent Optical pH Sensors with Minimal Effects of Ionic Strength. Ph. D. Thesis, Publikationsserver der Universitat Regensburg, Regensburg, Germany, 2004.

16. Tusa, J.K.; Leiner, M.J.P. Optodes Fluorescentes Pour Analytes de L'urgence. In Proceedings of the Annales de Biologie Clinique, Dossier: 2e Symposium International 'Gazomtrie Sanguine, Biocapteurs et Mthodes Optiques', Rennes, France, 30-31 May 2002. 
17. Povrozin, Y.A.; Markova, L.I.; Tatarets, A.L.; Sidorov, V.I.; Terpetschnig, E.A.; Patsenker, L.D. Near-infrared, dual-ratiometric fluorescent label for measurement of pH. Anal. Biochem. 2009, 390, 136-140.

18. Vasylevska, A.S.; Karasyov, A.A.; Borisov, S.M.; Krause, C. Novel coumarin-based fluorescent $\mathrm{pH}$ indicators, probes and membranes covering a broad $\mathrm{pH}$ range. Anal. Bioanal. Chem. 2007, 387, 2131-2141.

19. Szabelski, M.; Guzow, K.; Rzeska, A.; Malicka, J.; Przyborowska, M.; Wiczk, W. Acidity of carboxyl group of tyrosine and its analogues and derivatives studied by steady-state fluorescence spectroscopy. J. Photochem. Photobiol. A Chem. 2002, 152, 73-78.

20. Li, L.; Lee, L.J. Photopolymerization of HEMA/DEGDMA hydrogels in solution. Polymer 2005, $46,11540-11547$.

21. Son, Y.K.; Jung, Y.P.; Kim, J.H.; Chung, D.J. Preparation and properties of PEG-modified PHEMA hydrogel and the morphological effect. Macromol. Res. 2006, 14, 394-399.

22. Tian, Y.; Su, F.; Weber, W.; Nandakumar, V.; Shumway, B.R.; Jin, Y.; Zhou, X.; Holl, M.R.; Johnson, R.H.; Meldrum, D.R. A series of naphthalimide derivatives as intra and extracellular $\mathrm{pH}$ sensors. Biomaterials 2010, 31, 7411-7422.

23. Lu, H.; Jin, Y.; Tian, Y.; Zhang, W.; Holl, M.R.; Meldrum, D.R. New ratiometric optical oxygen and $\mathrm{pH}$ dual sensors with three emission colors for measuring photosynthetic activity in cyanobacteria. J. Mater. Chem. 2011, 21, 19293-19301.

24. Ferrari, L.; Fabbri, P.; Rovati, L.; Pilati, F. Photobleaching Effects in Organic Thin Film Sensing Probes. In Proceedings of the 2012 IEEE International Instrumentation and Measurement Technology Conference, Graz, Austria, 13-16 May 2012; pp. 1235-1239.

25. Klonis, N.; Sawyer, W.H. Spectral properties of the prototropic forms of fluorescein in aqueous solution. J. Fluorescence 1996, 6, 147-157.

(c) 2013 by the authors; licensee MDPI, Basel, Switzerland. This article is an open access article distributed under the terms and conditions of the Creative Commons Attribution license (http://creativecommons.org/licenses/by/3.0/). 